\title{
Establishing Point Correspondence Using Multidirectional Binary Pattern for Face Recognition
}

\author{
Sanqiang Zhao and Yongsheng Gao \\ School of Engineering, Griffith University, Nathan Campus, QLD 4111, Australia \\ \{s.zhao,yongsheng.gao\}@griffith.edu.au
}

\begin{abstract}
This paper presents a new Multidirectional Binary Pattern (MBP) for face recognition. Different from most Local Binary Pattern (LBP) related approaches which cluster LBP occurrences from whole image or partitioned subimage patches and use single or concatenated histogram measurement for recognition, $M B P$ is applied on a sparse set of shape-driven points. The new representation is designed for describing both global structure and local texture, and also significantly reduces the high dimensionality of LBP histogram description. Composed of binary patterns from multiple directions, $M B P$ is capable of extracting more discriminative features than LBP. The experiments on face recognition demonstrated the effectiveness of the proposed algorithm against expression and lighting variations.
\end{abstract}

\section{Introduction}

Local Binary Pattern (LBP) [1] has received noticeable attention in the face analysis community. As a non-parametric local descriptor, LBP was originally designed for texture description [2], but later extended to face recognition and outperformed existing methods such as PCA, LDA and EBGM. Two most important properties of the LBP operator in real-world applications are its computational efficiency and robustness against monotonic gray-level changes. The former property makes it possible to analyze images in challenging real-time settings. LBP has also been applied to other research areas such as facial expression analysis [3] and background modeling [4].

The basic principle of LBP is that a face can be seen as a composition of micro-patterns generated by the concatenation of the circular binary gradients. The statistical distribution (histogram) of these illumination invariant micro-patterns is used as a discriminative feature for identification. However, the LBP oper- ator is, by design, suitable for modeling repetitive texture patches, and is therefore sensitive to random noise in uniform image areas. Considering that a holistic LBP histogramming retains only the frequencies of micro-patterns and discards all information about their spatial layout, Ahonen et al. [1] employed a spatially enhanced histogram for face recognition, which is extracted from evenly divided sub-regions of a face, followed by a histogram concatenation. This arbitrary spatial partition is not in accordance with local facial topology, and leads to loss of discriminative power.

In this paper, we propose to extract binary patterns from the neighborhoods of a sparse set of shape-driven points which are detected from edge map with rich information content on a face image. Both the number and the locations of the points vary with different individuals such that diverse facial characteristics of these individuals can be represented. To enhance the discriminative power of binary patterns, we also propose a Multidirectional Binary Pattern (MBP) to reflect binary patterns spanning multiple directions. The new representation is capable of describing both global structure and local texture, and also significantly reduces the high dimensionality of LBP histogram description. It inherits most of the other advantages of LBP such as computational efficiency and exemption from training. Besides, the proposed method can effectively alleviate the problem of sensitivity to random noise in uniform image areas, because MBP features are only extracted from the neighborhoods of the sparse points, which are generally non-uniform areas. Using a new MBP measurement, we performed an investigation and evaluation of the proposed method on publicly available AR face database [5]. A higher recognition accuracy than that of Directional Corner Point (DCP) [6] was obtained in our experiments, demonstrating the validity of this method on face recognition.

In the following, Section 2 and 3 present the proposed MBP representation and measurement respec- 
tively. Section 4 provides comparative experiments on AR database. The last section concludes the paper.

\section{Multidirectional Binary Pattern}

\subsection{Local Binary Pattern}

The LBP operator marks each pixel $I_{c}$ of an image as a decimal number $L B P_{P, R}\left(I_{c}\right)$, which is formed by thresholding the $P$ equally spaced neighbor pixels $I_{p, R}(p=0, \cdots, P-1)$ on a circle of radius $R$ with the center pixel $I_{c}$ and concatenating the results binomially with factor $2^{p}$ :

$$
L B P_{P, R}=\sum_{p=0}^{P-1} T\left(I_{p, R}-I_{c}\right) 2^{p},
$$

where the thresholding function $T(x)$ is defined as

$$
T(x)=\left\{\begin{array}{ll}
1, & x \geq 0 \\
0, & x<0
\end{array} .\right.
$$

\subsection{Sparse Points Detection}

A sparse set of points representing the geometric structure are detected at positions which have rich edge information in a face image. The number of the sparse points and their locations can vary in order to better represent diverse facial characteristics of different individuals, such as dimples, moles, etc. We choose an edge detector from [7], followed by the Dynamic Two-Strip algorithm (Dyn2S) [8] to obtain these points. Fig. 1 illustrates two examples of sparse points superimposed on the original face images.
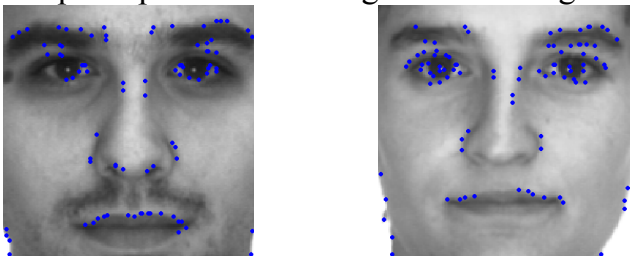

Fig. 1. Detected sparse points.

\subsection{Multidirectional Binary Pattern}

After the sparse points are detected, MBPs are extracted from these point positions. A MBP is defined as a pattern set which consists of four bunches of directional binary patterns: Horizontal Binary Patterns (HBPs), Vertical Binary Patterns (VBPs), Ascending Binary Patterns (ABPs) and Descending Binary Patterns (DBPs). In other words, MBP is composed of binary pattern bunches collected from four different directions. Fig. 2 visually illustrates the positions covered by these four pattern bunches. Similar to LBP, the pixels in the neighborhood are thresholded with the value of the center pixel, and then linearly concate- nated into four directional binary patterns as a local descriptor. One difference between MBP and LBP is that MBP is kept as original binary patterns, without being transformed into a decimal number for histogramming as is in LBP. It should be noted that although the four bunches of directional binary patterns may be derived from the same pixels, the pattern-level features they represent are different. This is demonstrated from the example in Fig. 3. Mathematically, a MBP set takes the form

$$
M B P=\left\{H B P_{L, N}, V B P_{L, N}, A B P_{L, N}, D B P_{L, N}\right\},
$$

where $H B P, V B P, A B P$ and $D B P$ refer to the four bunches of directional binary patterns respectively, with each bunch containing $N$ binary patterns of the length $L$. For instance, the bunch of HBPs can be represented as

$$
H B P_{L, N}=\left\{H B P_{L, 1}, H B P_{L, 2}, \cdots, H B P_{L, N}\right\},
$$

where each HBP is composed of concatenated $L$ binary values:

$$
H B P_{L, n}=\left[T\left(I_{H(1, n)}-I_{c}\right), T\left(I_{H(2, n)}-I_{c}\right), \cdots, T\left(I_{H(L, n)}-I_{c}\right)\right] .
$$

Here $I_{H(l, n)}(1 \leq l \leq L, 1 \leq n \leq N)$ represent the horizontally spaced pixels located at $L \times N$ positions in the neighborhood of the center pixel $I_{c}$ (see Fig. 2a). Similar representations are applied to the remaining three bunches of directional binary patterns. Fig. 3 provides an example of obtaining two bunches of directional binary patterns $H B P_{3,3}$ and $V B P_{3,3}$ with the parameters $L=3$ and $N=3$.

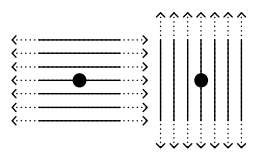

(a) (b)

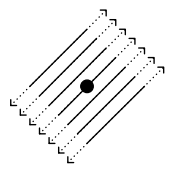

(c)

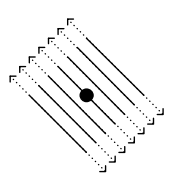

(d)

\begin{tabular}{|c|c|c|c|c|c|c|c|}
\hline 14 & 12 & 5 & \multirow{2}{*}{$\stackrel{\text { Threshold }}{\longrightarrow}$} & 1 & 0 & 0 & \multirow[b]{3}{*}{$V B P_{33}=\{110,011,010\}$} \\
\hline 17 & 14 & 20 & & 1 & 1 & 1 & \\
\hline 9 & 16 & 7 & & 0 & 1 & 0 & \\
\hline
\end{tabular}

Fig. 2. Multidirectional Binary Pattern (MBP). (a) HBP. (b) VBP. (c) ABP. (d) DBP. The black dot is the center pixel. The dashed arrows represent the bit-wise directional shifting.

Fig. 3. An illustration of obtaining $\boldsymbol{H B P}_{3,3}$ and $V B P_{3,3}$.

\section{Point Correspondence from MBPs}

\subsection{Binary Pattern Distance}

As a preliminary step, two distances are proposed to take measurement of two binary patterns (a model pattern $B P^{M}$ and a test pattern $B P^{T}$ ): pattern distance and shifting distance. Representing the pattern-level 
disparity between two binary patterns, the pattern distance $d_{p}$ is measured by examining the Hamming distances of the model pattern and the test pattern with several bit-wise shifts. The minimal value of these distances is selected as $d_{p}$. The shifting distance $d_{s}$ is defined as the number of shifting bits at which the pattern distance reaches the minimum. Fig. 4 provides examples of the proposed two distances. It is possible to assume that the pattern-level disparity originates from inter-class variation and the bit-wise shifting comes from intra-class variation. Therefore, $d_{p}$ and $d_{s}$ have the ability to reveal the local feature's interclass and intra-class variations respectively.

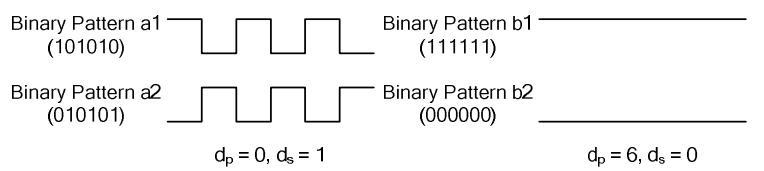

Fig. 4. The pattern distance $\left(d_{p}\right)$ and the shifting distance $\left(d_{s}\right)$.

Following the definitions of $d_{p}$ and $d_{s}$, the Binary Pattern Distance (BPD) of binary patterns $B P^{M}$ and $B P^{T}$ is represented as:

$$
B P D\left(B P^{M}, B P^{T}\right)=\sqrt{d_{p}^{2}+\rho d_{s}^{2}},
$$

where

$$
\left\{\begin{array}{l}
d_{p}=\min _{-K \leq k \leq K}\left\{H D\left(B P^{M}, S H\left(B P^{T}, k\right)\right)\right\} \\
d_{s}=\left|\underset{-K \leq k \leq K}{\arg \min }\left\{H D\left(B P^{M}, S H\left(B P^{T}, k\right)\right)\right\}\right|
\end{array} .\right.
$$

Here $\rho$ is used to balance the contributions of $d_{p}$ and $d_{s}$. HD stands for the Hamming distance. The operation $S H\left(B P^{T}, k\right)$ performs a bit-wise directional shifting on $B P^{T}$ for $k(k=-K, \cdots, 0, \cdots, K)$ times. A positive $k$ stands for a forward-shifting, a negative $k$ stands for a backward-shifting, and when $k$ equals 0 , no shifting operation is performed. $K(K \geq 0)$ is the bit-wise shifting limit.

\subsection{MBP Distance}

For two MBPs $\left(M B P^{M}\right.$ and $\left.M B P^{T}\right)$, the average BPD in each directional bunch is calculated, and then the minimal mean of four bunches is selected and defined as the MBP distance:

$$
\begin{aligned}
& d\left(M B P^{M}, M B P^{T}\right)=\min \left\{1 / N \sum_{n=1}^{N} B P D\left(H B P_{L, n}^{M}, H B P_{L, n}^{T}\right),\right. \\
& 1 / N \sum_{n=1}^{N} B P D\left(V B P_{L, n}^{M}, V B P_{L, n}^{T}\right), 1 / N \sum_{n=1}^{N} B P D\left(A B P_{L, n}^{M}, A B P_{L, n}^{T}\right), \\
& \left.1 / N \sum_{n=1}^{N} B P D\left(D B P_{L, n}^{M}, D B P_{L, n}^{T}\right)\right\} .
\end{aligned}
$$

This measurement involves bit-wise shifting of local patterns in four different directions (see dashed arrows in Fig. 2). By using a small balancing factor $\rho$, it can provide robustness to small local feature distortion caused by intra-class variation.

\subsection{Establishing Point Correspondence}

Motivated by a variant of Hausdorff distance [6, 9], a cost function is defined to find correspondence of MBP pairs between two face images. Given two finite MBP sets $M=\left\{M B P_{1}^{M}, M B P_{2}^{M}, \cdots, M B P_{P}^{M}\right\}$ representing a model face and $T=\left\{M B P_{1}^{T}, M B P_{2}^{T}, \cdots, M B P_{Q}^{T}\right\}$ representing a test face, where $P$ and $Q$ are the numbers of MBPs in $M$ and $T$ respectively. The cost function takes the form:

$$
D(M, T)=\max \{\operatorname{dir} D(M, T), \operatorname{dir} D(T, M)\},
$$

where the function $\operatorname{dir} D(M, T)$ is called the directed cost function from set $M$ to $T$. Since the point position $(x, y)$ where each MBP is extracted has been recorded, the directed MBP cost function is defined as

$$
\operatorname{dir} D(M, T)=\frac{1}{P} \sum_{p=1}^{P} \min _{1 \leq q \leq Q} \sqrt{\begin{array}{l}
\left(x_{p}^{M}-x_{q}^{T}\right)^{2}+\left(y_{p}^{M}-y_{q}^{T}\right)^{2} \\
+\lambda d^{2}\left(M B P_{p}^{M}, M B P_{q}^{T}\right)
\end{array}} .
$$

This is a compound measurement composed of both structural and textural information. The weight $\lambda$ balances the contributions of Euclidean distance and MBP distance. The cost function $D(M, T)$ evaluates the degree of mismatch between two MBP sets by measuring the distance of the MBP of $M$ that has the largest distance from any MBP of $T$, and vice versa.

\section{Experimental Results}

The proposed method is assessed on the publicly available AR face database [5], which contains 120 complete set of images ( 65 men and 55 women). All the images are normalized and cropped to $160 \times 160$ pixels based on the manually labeled positions of two eyes. Two examples of the cropped faces are illustrated in Fig. 1. We fix the MBP size as $L=8, N=8$, the bit-wise shifting limit as $K=4$ and the balancing factor as $\rho=0.1$ in our experiments.

\subsection{Determining $\lambda$}

In Equation (10), the weight $\lambda$ balances the contributions of structural and textural measurements. To determine $\lambda$, a series of preliminary experiments is performed using all the neutral expression faces in AR database. The model set is the neutral faces of the first session, and the test set is those of the second session. The top-one recognition rate against the weight $\lambda$ is displayed in Fig. 5. From the figure, it is found that the recognition rate reaches maximum when $\lambda=160$. The weight $\lambda=160$ is used in the rest of the experiments. 
In the following, the MBP method is compared with the DCP method [6] under various situations, using the neutral faces in normal condition taken in the first session as the model set.

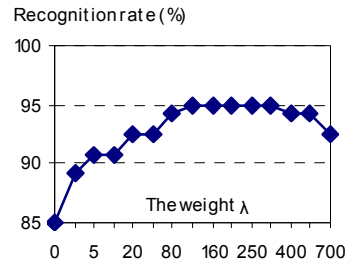

Fig. 5. Recognition rate against the weight.

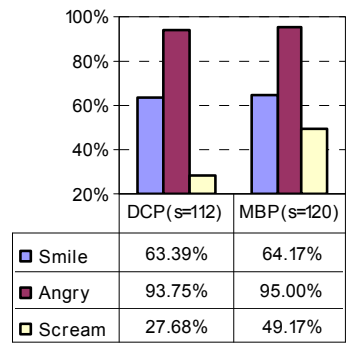

Fig. 7. Recognition rates under different expressions.

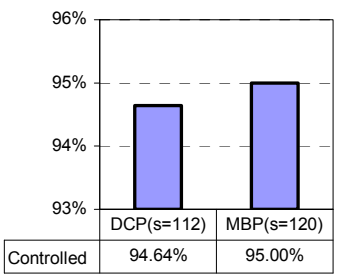

Fig. 6. Recognition rates under controlled condition.

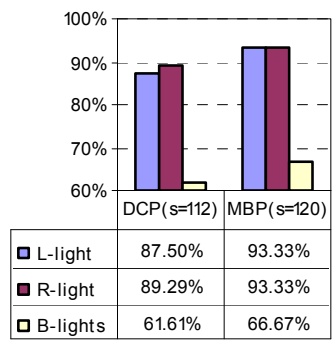

Fig. 8. Recognition rates under different illuminations.

\subsection{Face recognition results}

The face images under controlled condition in the second session are first used to evaluate the proposed method. The comparative recognition accuracy is illustrated in Fig. 6. Although the number of subjects used in this paper (120) is more than that in DCP (112), MBP still outperforms DCP.

To compare the performance with expression variations, the experiment is also performed on three different sets of images with smiling, angry and screaming expressions in the first session. The results listed in Fig. 7 show that the performance of MBP is much better than DCP under all three expression variations, especially under the screaming condition, where the improvement is over $20 \%$. This can be explained by the robustness of MBP against local feature distortion.

We finally perform the experiment under three different conditions of illumination change: left light, right light and both lights on. Fig. 8 displays these experimental results. The recognition accuracy of MBP is noticeably above $90 \%$ when either left or right light on. This demonstrates that MBP is very tolerant to lighting changes. However, it is still sensitive to extreme lighting, which causes strong specular reflectance on the face skin and thus could erase some sparse points.

\section{Conclusions}

This paper proposes a new Multidirectional Binary Pattern (MBP) for face representation. Faces are modeled as a sparse set of shape-driven points with MBP attached on each point. The main contributions of the proposed method are: 1) Binary pattern bunches from multiple directions are collected to enhance the discriminative power of local features. 2) In stead of histogramming all the pixels of an image, local features are extracted from sparse points to reduce the storage demand. 3) A specially designed MBP measurement is proposed to evaluate binary patterns and establish point correspondence for face recognition. This study demonstrates that the proposed MBP method provides a new solution towards robust face recognition.

\section{Acknowledgement}

This research was supported by the Australian Research Council (ARC) Discovery Grants DP0451091 and DP0877929.

\section{References}

[1] T. Ahonen, A. Hadid, and M. Pietikäinen, "Face Description with Local Binary Patterns: Application to Face Recognition," TPAMI, 28(12): 2037-2041, 2006.

[2] T. Ojala, M. Pietikäinen, and T. Mäenpää, "Multiresolution Gray-Scale and Rotation Invariant Texture Classification with Local Binary Patterns," TPAMI, 24(7): 971987, 2002.

[3] G. Zhao and M. Pietikäinen, "Dynamic Texture Recognition Using Local Binary Patterns with an Application to Facial Expressions," TPAMI, 29(6): 915-928, 2007.

[4] M. Heikkilä and M. Pietikäinen, "A Texture-Based Method for Modeling the Background and Detecting Moving Objects," TPAMI, 28(4): 657-662, 2006.

[5] A.M. Martinez and R. Benavente, "The AR Face Database," CVC Technical Report \#24, June 1998.

[6] Y. Gao and Y. Qi, "Robust Visual Similarity Retrieval in Single Model Face Databases," Pattern Recognition, 38(7): 1009-1020, 2005.

[7] R. Nevatia and K.R. Babu, "Linear Feature Extraction and Description," CGIP, 13(3): 257-269, 1980.

[8] M.K. Leung and Y.-H. Yang, "Dynamic Two-Strip Algorithm in Curve Fitting," Pattern Recognition, 23(1-2): 69-79, 1990.

[9] S. Zhao and Y. Gao, "Significant Jet Point for Facial Image Representation and Recognition," Proceedings of ICIP, 2008. 